\title{
Investigation of the Characteristics of the Electrodynamic Wheel Suspension Device with two-DOF Motion
}

\author{
Jiaheng Duan, Wenlong Zhang, Song Xiao, Kunlun Zhang, and J. K. Sykulski, Fellow, IEEE
}

\begin{abstract}
A three-dimensional (3-D) derivation of an analytical model for an electrodynamic wheel suspension (EDWS) device is proposed in this paper. The equations for source field generated by a Halbach wheel are derived by the superposition principle of magnetic charge sheets. The eddy current field distribution within the semicircular pipe track is modeled using the second-order potential (SOVP) theory. The analytical models describing fields and forces are validated by both finite element simulations and experimental results. In addition, the characteristics of the EDWS device with two-degree-of-freedom (DOF) motions are studied based on the proposed equations.
\end{abstract}

Index Terms-Electrodynamic suspension, electromagnetic launcher, magnetic levitation (MAGLEV).

\section{INTRODUCTION}

$\mathrm{T}$ HE simultaneous rotational and translational motion of permanent magnets (PMs) above a conducting plate create velocity dependent lift and drag forces; this so-called electrodynamic suspension (EDS) system has been widely used in high-speed maglev transportation as well as in electromagnetic launchers due to its excellent performance, in particular self-stability and simplicity [1].

In the past few years, a variety of EDS topologies with rotating Halbach rotor have been developed and investigated [2]-[5]. For instance, a partial overlap type magnet wheel was proposed in [4] to achieve a self-driven function for the EDS device. More recently, an electrodynamic wheel topology integrated suspension and propulsion for vehicles was numerically studied [5]. However, it is hard to achieve a relatively high lift-to-weight ratio for the electrodynamic wheel concept due to the low utilization of the PM source field.

Manuscript received November 10, 2019; revised April 28, 2020; accepted ? ??. Date of publication May ?? ??, 2020; date of current version ?? ??, 2019. This work was supported by the National Natural Science Foundation of China (Grant No. 51707166). The review of this paper was arranged by Senior Editor ??. ????. (Corresponding author: Jiaheng Duan.).

J. Duan, W. Zhang, S. Xiao and K. Zhang are with The Key Laboratory of Magnetic Suspension Technology and Maglev Vehicle, Ministry of Education and School of Electrical Engineering, Southwest Jiaotong University, Chengdu 610031, China (e-mail: duanjh@my.swjtu.edu.cn).

J. K. Sykulski is with Electronics and Computer Science, University of Southampton, SO17 1BJ, UK (e-mail: jks@soton.ac.uk).

Color versions of one or more of the figures in this paper are available online at http://ieeexplore.ieee.org.

Digital Object Identifier (inserted by IEEE).

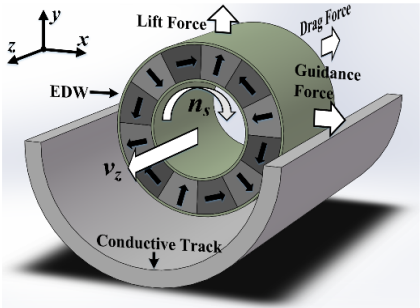

(a)

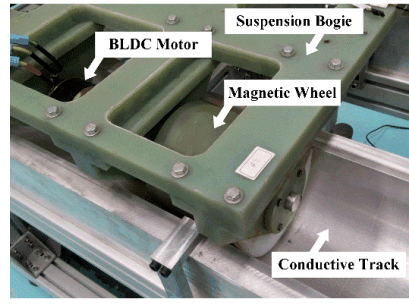

(b)
Fig. 1. (a) 3-D schematic of the EDWS device; (b) experimental setup

In our previous work [6], a novel electrodynamic wheel suspension (EDWS) device consisting of a Halbach PM rotor and semicircular pipe track was proposed for enhancing the lift-to-weight ratio. In this approach, a static levitation for the vehicle can be achieved without translational velocity.

A 2-D magnetic vector potential-based analytical method was proposed in [6] for evaluating the performances of the novel topology. However, since the axial width of the PM rotor was neglected in the derived equations, the MVP-based method was found to be highly inaccurate compared with the time-consuming 3-D finite element method [7]. Moreover, the 2-D analytical method is inappropriate for the case when the rotor is moving translationally above the track.

In this paper, analytical-based equations for describing the eddy current field, three-dimensional (3-D) electromagnetic forces and power loss generated by an EDWS device are derived for the case where the rotor is operating with rotational and/or translational velocities, as illustrated in Fig. 1 (a). An experimental setup was constructed as shown in Fig. 1 (b). The magnetic Halbach rotor is driven by a brushless direct current (BLDC) motor; both the rotor and the driving motor are installed on the suspension bogie. The semicircular track is made of conducting material and the air-gap between the rotor and the track is adjustable for the verification of an analytical model under various conditions.

\section{SOURCE FIELD}

The charge model of a single curve-shaped PM with positive diametrical direction parallel magnetization is selected as an example, as shown in Fig. 2 (a). The equation for describing the source field can be derived by the sum of four charge sheets [8], [9] (sI S4). The corresponding magnetostatic equations for this problem are: 


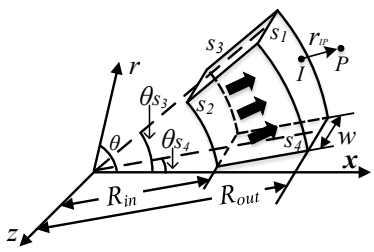

(a)

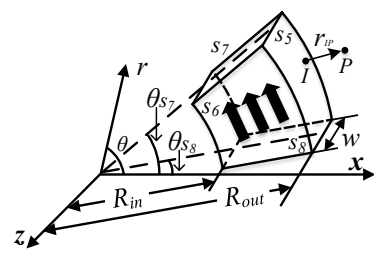

(b)
Fig. 2. (a) Magnetic charge sheets of one single curve-shaped PM with diametrical magnetization; (b) magnetic charge sheets of one single curve-shaped PM with tangential magnetization

$$
\begin{gathered}
\nabla \cdot \mathbf{B}=\rho_{s} \\
\mathbf{B}=-\mu_{0} \nabla \varphi
\end{gathered}
$$

where $\rho_{s}$ is the corresponding magnetic charge density which is related to the remanence $\left(B_{r}\right)$ of the PM. $\varphi$ is the magnetic scalar potential in the cylindrical coordinate system, which can be solved by substituting (2) into (1)

$$
\varphi^{s_{n}}=\frac{1}{4 \pi \mu_{0}} \int_{s_{n}} \frac{\rho_{s_{n}}}{r_{P I}} d S
$$

where $r_{P I}$ is the distance between the space point $P(r, \theta, z)$ and the source point $I\left(r_{0}, \theta_{0}, z_{0}\right)$, and $r_{P I}$ is given by

$$
r_{P I}^{2}=\left[r \cos (\theta)-r_{0} \cos \left(\theta_{0}\right)\right]^{2}+\left[r \sin (\theta)-r_{0} \sin \left(\theta_{0}\right)\right]^{2}+\left(z-z_{0}\right)^{2}
$$

Solving (2) with a combination of (3) and (4), the analytical model of one charge sheet can be obtained. For charge sheets $s I$ and $s_{2}$, the equation of the flux density along radial direction can be expressed as

$$
\begin{aligned}
B_{r}^{s_{n}}\left(r, \theta, z, z_{0}\right) & =-\mu_{0} \frac{\partial \varphi^{s_{n}}}{\partial r}=\frac{R_{s_{n}}}{4 \pi} \int_{\theta_{s 4}}^{\theta_{s 3}} \rho_{s_{n}}\left[\int \frac{\partial\left(r_{P I}^{-1}\right)}{\partial r} d z_{0}\right] d \theta_{0} \\
& =\frac{R_{s_{n}}}{4 \pi} \int_{\theta_{s 4}}^{\theta_{s 3}} \frac{\rho_{s_{n}}\left[r-R_{s_{n}} \cos \left(\theta-\theta_{0}\right)\right]\left(z-z_{0}\right)}{r_{P I}\left[r^{2}+R_{s_{n}}^{2}-2 R_{s_{n}} r \cos \left(\theta-\theta_{0}\right)\right]} d \theta_{0}
\end{aligned}
$$

where

$$
\left\{\begin{array}{l}
\rho_{s_{n}}=B_{r} \cdot \cos \left[\theta_{0}-\left(\theta_{s_{3}}+\theta_{s_{4}}\right) / 2\right] \quad n=1,2 \\
R_{s_{1}}=R_{\text {out }}, R_{s_{2}}=R_{\text {in }}
\end{array}\right.
$$

For charge sheets $s_{3}$ and $s_{4}$, the equation of the flux density along the radial direction is given by

$$
\begin{aligned}
B_{r}^{s_{n}}\left(r, \theta, z, z_{0}\right) & =-\mu_{0} \frac{\partial \varphi^{s_{n}}}{\partial r}=\frac{R_{s_{n}}}{4 \pi} \int_{R_{\text {in }}}^{R_{\text {out }}} \rho_{s_{n}}\left[\int \frac{\partial\left(r_{P I}^{-1}\right)}{\partial r} d z_{0}\right] d r_{0} \\
& =\frac{\rho_{s_{n}}}{4 \pi} \int_{R_{\text {in }}}^{R_{\text {out }}} \frac{\left[r-r_{0} \cos \left(\theta_{s_{n}}-\theta\right)\right]\left(z-z_{0}\right)}{r_{P I}\left[r^{2}+r_{0}^{2}-2 r_{0} r \cos \left(\theta_{s_{n}}-\theta\right)\right]} d r_{0}
\end{aligned}
$$

where

$$
\rho_{s_{n}}=B_{r} \cdot \sin \left[\theta_{s_{n}}-\left(\theta_{s_{3}}+\theta_{s_{4}}\right) / 2\right] \quad n=3,4
$$

Substituting the limits of $z_{0}$ into (5) and (7), the equation describing the flux density of a curve-shaped PM with positive diametrical direction parallel magnetization can be obtained by superposition of charge sheets $s 1 \sim S 4$, which is defined as

$$
B_{r}^{\text {radial }}(r, \theta, z)=\sum_{n=1}^{4}\left(B_{r}^{s_{n}}\left(r, \theta, \frac{w}{2}\right)-B_{r}^{s_{n}}\left(r, \theta,-\frac{w}{2}\right)\right)
$$

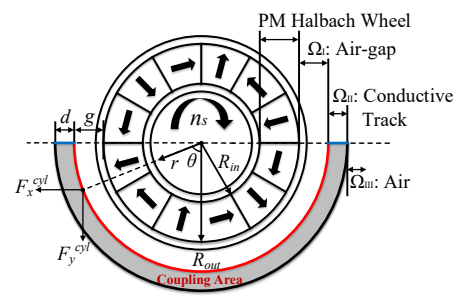

(a)

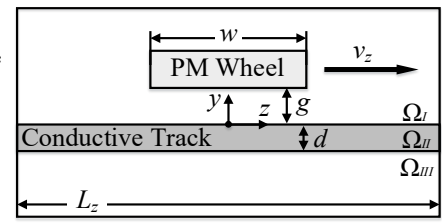

(b)

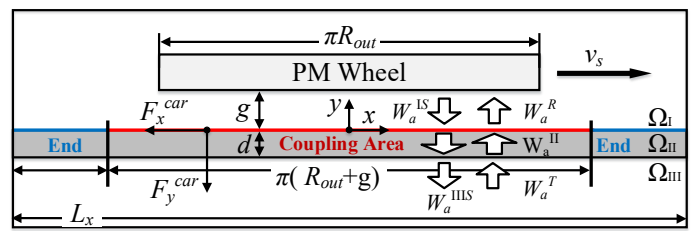

(c)

Fig. 3. (a) 2-D layer model in the cylindrical coordinate system (b) $y-z$ and (c) $x-y$ view of solution domain in the Cartesian coordinate system

The analytical model of a curve-shaped PM with a positive tangential direction parallel magnetization, as shown in Fig. 2 (b), can also be defined by given equations. Equations (5) and (7) are available for charge sheets $s_{5} \sim s_{8}$ by changing the limits of integration and replacing charge density with

$$
\begin{array}{lc}
\rho_{s_{n}}=B_{r} \cdot \sin \left[\theta_{0}-\left(\theta_{s_{7}}+\theta_{s_{8}}\right) / 2\right] & n=5,6 \\
\rho_{s_{n}}=B_{r} \cdot \cos \left[\theta_{s_{n}}-\left(\theta_{s_{7}}+\theta_{s_{8}}\right) / 2\right] & n=7,8
\end{array}
$$

After substituting (10) and (11) into (5) and (7), respectively, the equation for flux density generated by a single curve-shaped PM with tangential magnetization, $B_{r}^{\text {axial }}$, can be found from (9).

Finally, the analytical equation for describing the source field of a PM rotor, as shown in Fig. 3 (a), can be expressed by a superposition of all the PMs in the solution domain [9] i.e.

$$
B_{r}^{S}(r, \theta, z)=\sum_{\text {All PMs }}\left[B_{r}^{\text {radial }}(r, \theta, z)+B_{r}^{\text {axial }}(r, \theta, z)\right]
$$

\section{EDDY CURRENT FIELD}

The cylindrical layer model of the EDWS device is shown in Fig. 3 (a). For simplification, the equivalent linear model in the Cartesian coordinate system can be established by applying the coordinate transformation. The equivalent model is assumed where the transverse end effect is negligible; moreover, there is no normal component in the eddy current vector. The $y-z$, as well as the $x-y$, views of the solution domain are shown in Fig. 3 (b) and (c), respectively.

\section{A. Conductive Region $\Omega_{\text {II }}$}

The problem of induced eddy current within a conductive plate can be formulated by the magnetic vector potential [10], $\mathbf{A}$, as follows

$$
\nabla^{2} \mathbf{A}=-\mu_{0} \gamma \mathbf{v} \cdot \nabla \mathbf{A}
$$

where $\gamma$ is conductivity and $\mathbf{v}$ is the velocity vector, while utilizing the MVP and the SOVP theory for solving the flux distribution within the conductive track

$$
\mathbf{B}=\nabla \times \mathbf{A}=\nabla \times \nabla \times \mathbf{W}
$$


where $\mathbf{B}$ is flux density, $\mathbf{W}$ is SOVP and can be decomposed into two scalar potentials with $y$-preferred direction [10], [11]

$$
\mathbf{W}=\vec{y} \cdot W_{a}+\vec{y} \times \nabla W_{b}
$$

where $W_{a}$ and $W_{b}$ represent transverse electric (TE) and transverse magnetic (TM) potentials, respectively. Since the assumption that there are no normal eddy currents, only the TE potential exists in the conducting region and the TM potential is zero.

In the conducting region, the SOVP of the eddy current field can be derived by substituting (15) and (14) into (13)

$$
\nabla^{2} W_{a}^{\mathrm{II}}=-\mu_{0} \gamma\left(v_{s} \frac{\partial W_{a}^{\mathrm{II}}}{\partial x}+v_{z} \frac{\partial W_{a}^{\mathrm{II}}}{\partial z}\right), \text { in } \Omega_{\mathrm{II}}
$$

where $v_{s}(\mathrm{~km} / \mathrm{h})$ is the equivalent translational velocity and is related to the rotational speed $n_{s}(\mathrm{rpm})$ by

$$
v_{s}=0.12 n_{s} \pi\left(R_{\text {out }}+g\right)
$$

By applying the separation of variables method to (14), the general solution for the TE potential can be found as

$$
W_{a}^{\mathrm{II}}(x, y, z)=\sum_{m=-\infty}^{+\infty} \sum_{n=-\infty}^{+\infty}\left(C_{m n}^{\mathrm{II}} e^{\alpha_{m n} y}+D_{m n}^{\mathrm{II}} e^{-\alpha_{m n} y}\right) e^{j \xi_{m} x} e^{j k_{n} z}
$$

where $C_{m n}^{\mathrm{II}}$ and $D_{m n}^{\mathrm{II}}$ are unknown coefficients, and

$$
\begin{gathered}
\xi_{m}=2 \pi m / L_{x} \\
k_{n}=2 \pi n / L_{z} \\
\alpha_{m n}=\sqrt{\xi_{m}^{2}+k_{n}^{2}-j \mu_{0} \gamma\left(v_{s} \xi_{m}+v_{z} k_{n}\right)}
\end{gathered}
$$

\section{B. Nonconductive Region $\Omega_{\mathrm{I}}$ and $\Omega_{\mathrm{III}}$}

The TE potential within the nonconducting regions $\Omega_{\mathrm{I}}$ and

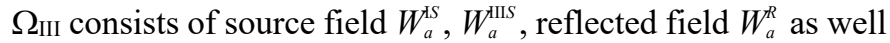
as transmitted field $W_{a}^{T}$, as shown in the equivalent linear model, Fig. 3 (c). Since the conductivity $\gamma$ in nonconducting regions is zero, (16) can be simplified to

$$
\nabla^{2} W_{a}=0, \text { in } \Omega_{\mathrm{I}} \& \Omega_{\mathrm{III}}
$$

Similarly, solving (22) via the separation of variables method, the TE potentials of reflected and transmitted field terms are derived as

$$
\begin{gathered}
W_{a}^{R}(x, y, z)=\sum_{m=-\infty}^{+\infty} \sum_{n=-\infty}^{+\infty} \frac{C_{m n}^{R}}{\kappa_{m n}^{2}} e^{j \xi_{m} x} e^{j k_{n} z} e^{-\kappa_{m n} y} \\
W_{a}^{T}(x, y, z)=\sum_{m=-\infty}^{+\infty} \sum_{n=-\infty}^{+\infty} \frac{C_{m n}^{T}}{\kappa_{m n}^{2}} e^{j \xi_{m} x} e^{j k_{n} z} e^{\kappa_{m n}(y+d)}
\end{gathered}
$$

where $C_{m n}^{R}$ and $C_{m n}^{T}$ are unknowns and

$$
\kappa_{m n}^{2}=\xi_{m}^{2}+k_{n}^{2}
$$

Substituting (22) into (14), it transpires that in the equivalent linear model the relationship between the $y$-component source flux density and the TE potential can be expressed as

$$
B_{y}^{S}=\frac{\partial^{2} W_{a}^{S}}{\partial y^{2}}
$$

In order to derive the expression for the TE potential, it can be seen from (26) that only the $y$-component of the source field on the surface of the conductive track is needed. Assuming the upper track surface is selected as the planar charge sheet [8], the
TABLE I

PARAMETERS

\begin{tabular}{llll}
\hline \hline Parameters & Unit & FEM & EXP \\
\hline Outer radius, $R_{\text {out }}$ & $\mathrm{mm}$ & 100 & 60 \\
Inner radius, $R_{\text {in }}$ & $\mathrm{mm}$ & 70 & 40 \\
Width of wheel, $w$ & $\mathrm{~mm}$ & 200 & 50 \\
Number of PMs, $N$ & - & 12 & 12 \\
Remanence, $B_{r}$ & Tesla & 1.25 & 1.19 \\
Air-gap, $g$ & $\mathrm{~mm}$ & 10 & $15 / 25$ \\
Thickness of track, $d$ & $\mathrm{~mm}$ & 20 & 20 \\
Conductivity, $\gamma$ & $\mathrm{S} / \mathrm{m}$ & $2.57 \mathrm{e} 7$ & $2.57 \mathrm{e} 7$ \\
\hline \hline
\end{tabular}

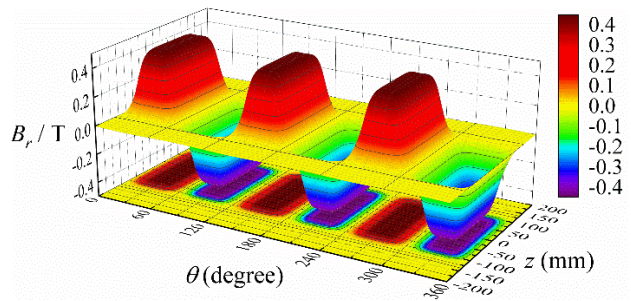

(a)

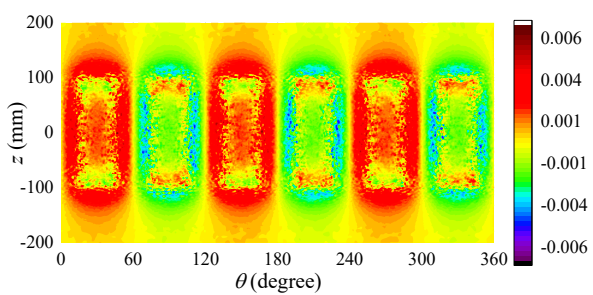

(b)

Fig. 4. (a) Flux density along the radial direction calculated by charge model; (b) calculation error between the analytical model and finite element model

$y$-component of the source field within the nonconductive region is calculated using

$$
B_{y}^{S}(x, y, z)=\sum_{m=-\infty}^{+\infty} \sum_{n=-\infty}^{+\infty} S_{m n} e^{j \xi_{m} x} e^{j k_{n} z} e^{k_{m m} y}, \quad \text { in } \Omega_{\mathrm{I}} \& \Omega_{\mathrm{III}}
$$

where $S_{m n}$ is the Fourier coefficient of source flux density on the selected charge sheet, which can be calculated via applying 2-D Fourier transform to (12), which yields

$$
S_{m n}=\frac{1}{L_{x} L_{z}} \int_{-L_{z} / 2}^{L_{z} / 2} \int_{-L_{x} / 2}^{L_{x} / 2} B_{r}^{s}\left(R_{\text {out }}+g, \frac{\pi x}{L_{x}}, z\right) e^{-j \xi_{m} x} e^{-j k_{n} z} d x d z
$$

Thus, within the air region, the TE potential due to the $y$-component source field, namely the radial component of the flux density in the cylindrical coordinate system, can be obtained by solving (26) with a combination of (27), as follows

$$
W_{a}^{S}(x, y, z)=\sum_{m=-\infty}^{+\infty} \sum_{n=-\infty}^{+\infty} \frac{S_{m n}}{\kappa_{m n}^{2}} e^{j \xi_{m} x} e^{j k_{n} z} e^{\kappa_{m n} y}
$$

\section{Boundary Condition and Unknown Coefficients}

The continuity boundary condition [11] is applied on both the upper and lower surfaces of the linear conductive track; the following equations of TE potential are therefore satisfied

$$
\begin{gathered}
W_{a}^{R}+W_{a}^{\mathrm{IS}}=W_{a}^{\mathrm{II}}, \quad \text { at } y=0 \\
\partial W_{a}^{R} / \partial y+\partial W_{a}^{\mathrm{IS}} / \partial y=\partial W_{a}^{\mathrm{II}} / \partial y, \quad \text { at } y=0 \\
W_{a}^{T}+W_{a}^{\mathrm{IIS}}=W_{a}^{\mathrm{II}}, \quad \text { at } y=-d \\
\partial W_{a}^{T} / \partial y+\partial W_{a}^{\mathrm{IIS}} / \partial y=\partial W_{a}^{\mathrm{II}} / \partial y, \quad \text { at } y=-d
\end{gathered}
$$




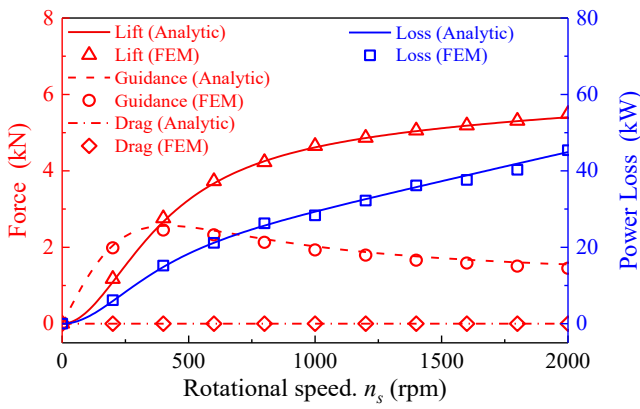

(a)

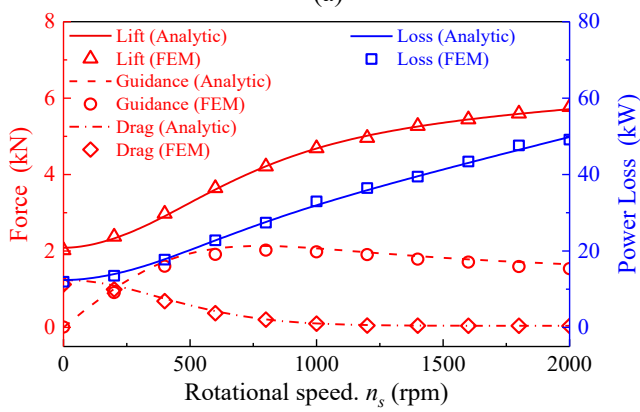

(b)

Fig. 5. (a) Lift, guidance force and loss as a function of $n_{s}$ for $v_{z}=0 \mathrm{~km} / \mathrm{h}$ and (b) $v_{z}=36 \mathrm{~km} / \mathrm{h}$

Substituting (18), (23), (24) and (29) into the boundary conditions (30)-(33) and canceling out common terms, the unknown coefficients of TE potential within region $\Omega_{\text {I I }}$ and $\Omega_{\text {III }}$ can be obtained

$$
\begin{gathered}
C_{m n}^{R}=\frac{S_{m n}\left(e^{\alpha_{m n} d}-e^{-\alpha_{m n} d}\right)\left(\kappa_{m n}^{2}-\alpha_{m n}^{2}\right)}{e^{\alpha_{m n} d}\left(\kappa_{m n}+\alpha_{m n}\right)^{2}-e^{-\alpha_{m n} d}\left(\kappa_{m n}-\alpha_{m n}\right)^{2}} \\
C_{m n}^{T}=\frac{4 S_{m n} \kappa_{m n} \alpha_{m n}}{e^{\alpha_{m n} d}\left(\kappa_{m n}+\alpha_{m n}\right)^{2}-e^{-\alpha_{m n} d}\left(\kappa_{m n}-\alpha_{m n}\right)^{2}}-S_{m n} e^{-\kappa_{m n} d}
\end{gathered}
$$

Substituting (15) and (22) into (14), the flux density B in nonconductive regions can be given by

$$
\mathbf{B}=\frac{\partial^{2} W_{a}}{\partial x \partial y} \vec{x}-\left(\frac{\partial^{2} W_{a}}{\partial x^{2}}+\frac{\partial^{2} W_{a}}{\partial z^{2}}\right) \vec{y}+\frac{\partial^{2} W_{a}}{\partial z \partial y} \vec{z}=\nabla\left(\frac{\partial W_{a}}{\partial y}\right)
$$

\section{Force AND POWER LosS}

In Fig. 3 (c), $F_{x}^{c a r}$ and $F_{y}^{c a r}$ are equivalent lift and drag forces in the Cartesian coordinate system, respectively. The Maxwell stress tensor is applied for calculating the distribution of force density on the conducting track, written as follows [11]

$$
\mathbf{f}^{C a r}(x, z)=\frac{1}{\mu_{0}} \operatorname{Re}\left[\begin{array}{l}
B_{y}^{\text {IS }}(x,-d, z)^{*} \mathbf{B}^{R}(x,-d, z) \\
-B_{y}^{\text {IIIS }}(x, 0, z)^{*} \mathbf{B}^{T}(x, 0, z)
\end{array}\right]
$$

Similarly, $F_{x}^{c y l}$ and $F_{y}^{c y l}$ are levitation and guidance forces on the track, respectively, as shown in Fig. 3 (a). Both the force vector and load torque on the magnetic rotor are evaluated as

$$
\mathbf{F}^{c y l}=\int_{-L_{z} / 2}^{L_{z} / 2} \int_{-R / 2}^{R / 2}\left(\begin{array}{ccc}
\cos (\pi x / R) & \sin (\pi x / R) & 0 \\
-\sin (\pi x / R) & \cos (\pi x / R) & 0 \\
0 & 0 & 1
\end{array}\right) \cdot \mathbf{f}^{C a r} d x d z
$$

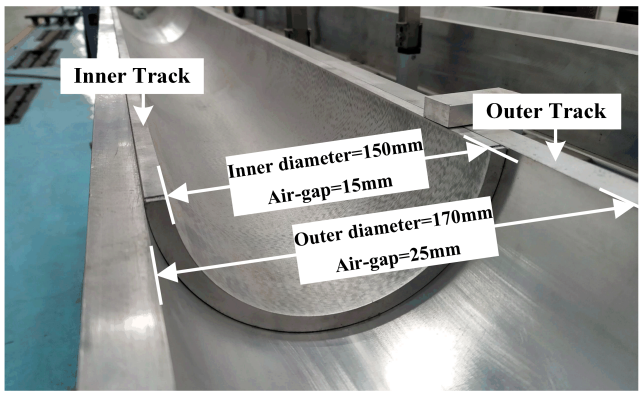

Fig. 6. Conductive track constructed with adjustable air-gap

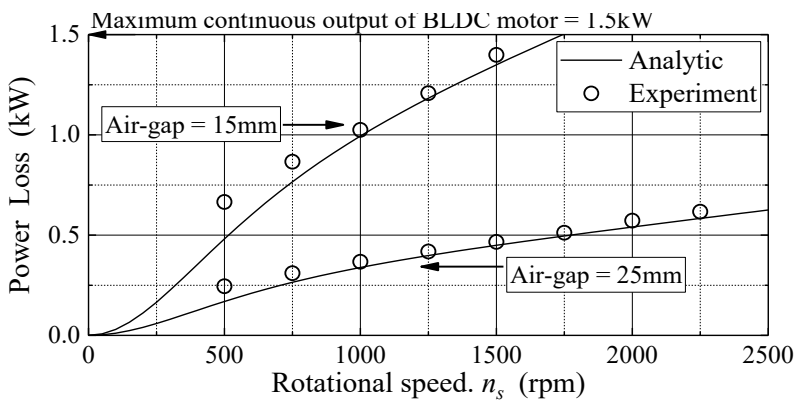

Fig. 7. Comparison between experimental and analytical results

$$
T_{e m}=\int_{-L_{z} / 2}^{L_{z} / 2} \int_{-R / 2}^{R / 2}\left(f_{x}^{\text {Car }} / R_{\text {out }}\right) d x d z
$$

where $R$ is the inner radius of the track, equal to the sum of $R_{\text {out }}$ and air-gap $g$. Using (38) and (39) the power loss of a single magnetic rotor can be written as

$$
P_{e m}=P_{e m 1}+P_{e m 2}=\left(T_{e m} \pi n_{s} / 30\right)+\left(F_{z}^{c l l} v_{z}\right)
$$

where $P_{e m l}$ is the power loss of the rotary suspension system and $P_{e m 2}$ is the power loss of the linear propulsion system.

\section{VALIDATION AND INVESTIGATION}

In order to validate the proposed method, both the finite element model (FEM) and experimental (EXP) setup have been constructed with the parameters defined as in Table I.

\section{A. Verification of source field}

Fig. 4 (a) shows the flux density along the radial direction $\left(B_{r}\right)$ generated by a Halbach PM wheel with three pole pairs, calculated by the proposed equations (5), (7) and (12). It can also be seen in Fig. 4 (b) that the maximum error of the calculation is less than $1.5 \%$.

\section{B. Forces and power loss}

In Fig. 5 (a), the forces and loss generated by a single magnetic rotor with one rotational DOF motion have been calculated by both the analytical and the FE method [12]. It is shown that the drag force $F_{z}$ is always zero since there is no translational velocity. Fig. 5 (b) shows the device performance when the rotor is moving translationally $\left(v_{z}=36 \mathrm{~km} / \mathrm{h}\right)$ above the track; it can be seen that following an increase of rotational speed, the drag force gradually declines.

In order to verify the proposed method experimentally [13], one single magnetic rotor on the experimental vehicle is shown in Fig. 1 (b). The conductive track has been constructed with an 


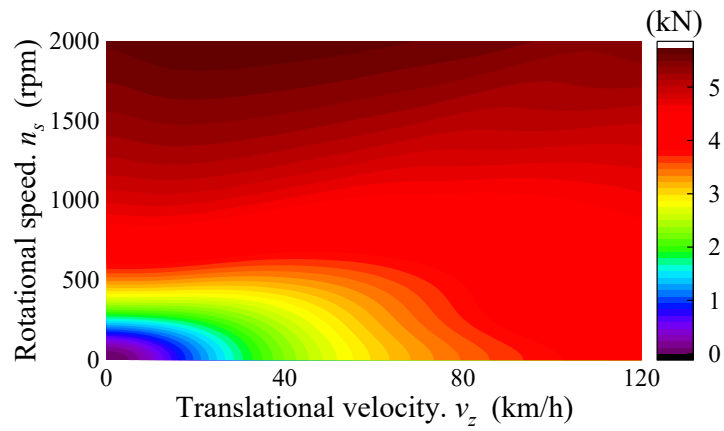

(a)

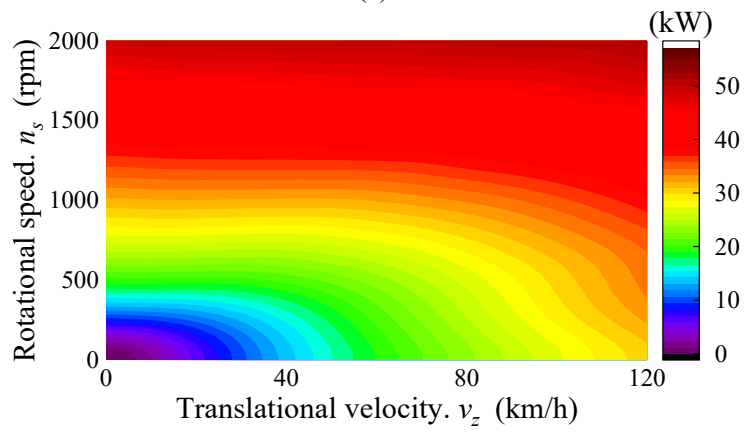

(b)

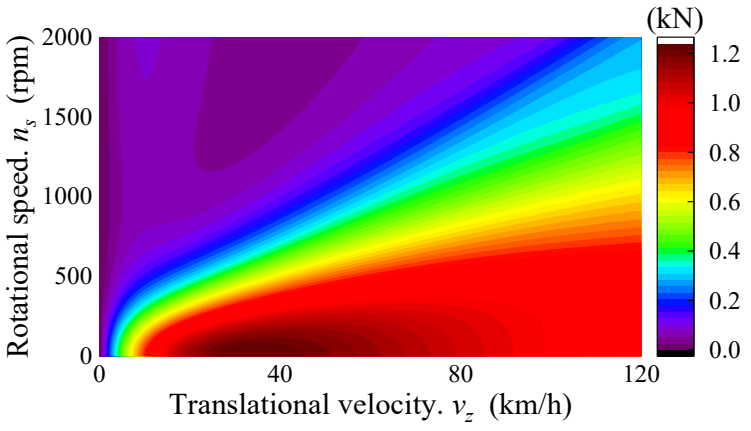

(c)

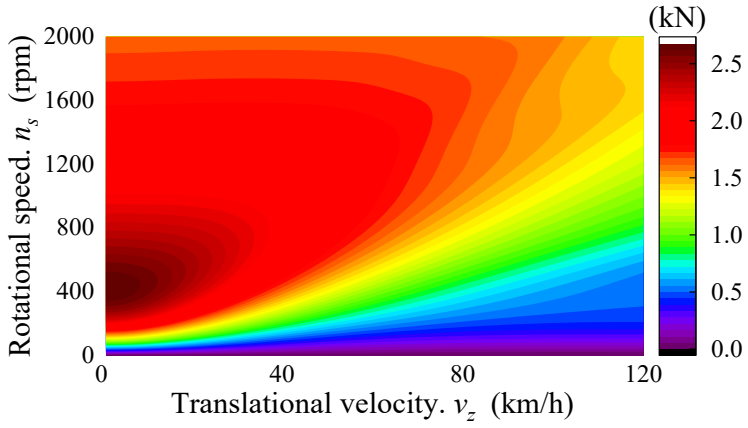

(d)

Fig. 8. (a) Lift force $(\mathrm{kN})$; (b) total power loss $(\mathrm{kW})$; (c) drag force $(\mathrm{kN})$ and (d) guidance force $(\mathrm{kN})$

adjustable air-gap, as shown in Fig. 6. The comparison of the results for two different air-gaps is shown in Fig. 7. Due to the non-negligible loss of driving motor and direct current power supply, especially the ohmic loss of BLDC motor under the constant torque operation, it is found that the experimental results are larger than the analytical results when the motor is running at low speed.
Since both Fig. 5 and Fig. 7 show good agreement between the analytical and the FE and the experimental methods, the validity of the proposed equations may be considered as verified.

\section{Investigation of performance with two-DOF motion}

The variation of the device performance with both translational and rotational velocities is shown in Fig. 8. It is seen from Fig. 8(a) and (b) that the lift force $F_{y}$ and the total loss $P_{e m}$ increases continuously with the rise of velocities $n_{s}$ and $v_{z}$.

It can be seen from Fig. 8 (c) that, in order to reduce the drag force $F_{z}$ as much as possible, a relatively high rotational speed is required of the magnetic rotor when the maglev vehicle operates at a high translational velocity.

Fig. 8 (d) shows the impact of velocities on the guidance force $F_{x}$. When $v_{z}$ is fixed at a certain value, it is noticed that as $n_{s}$ goes up, the value of $F_{x}$ increases initially, then gradually declines.

Overall, in order to ensure the maglev vehicle running within a reasonable operating region [14], a control trajectory for velocities could be designed based on the characteristics of the proposed EDWS system.

\section{CONCLUSION}

A methodology for an EDWS device with two-DOF motion has been derived in this paper. The validity of the generalized method is verified both numerically and experimentally, with the average error between the FE and the proposed method maintained at around 3\%. The steady-state performance of the EDWS device is studied analytically via the proposed model. Moreover, the characteristics of a single magnetic rotor with translational and/or rotational velocities have been investigated based on the proposed equations. The investigation showed a coupling relationship between the device performance and the two DOF motions.

\section{REFERENCES}

[1] Jacobs W A. "Magnetic launch assist-NASA's vision for the future," IEEE Trans. Magn., Vol.37, no.1, pp. 55-57, 2001.

[2] H. W. Cho, H. W. Han, J. S. Bang, et al. "Characteristic analysis of electrodynamic suspension device with permanent magnet Halbach array," Journal of Applied Physics, Vol.105, no.1, pp.501, 2009.

[3] A. Musolino, M. Raugi, R. Rizzo, et al. "Stabilization of a permanent-magnet MAGLEV system via null-flux coils," IEEE Trans. Plasma Sci., vol45, no.5, pp. 1242-1247, 2015.

[4] N. Fujii, S. Nonaka, G. Hayashi. "Design of magnet wheel integrated own drive," IEEE Trans. Magn, vol35, no.5, pp. 4013-4015, 1999.

[5] J. Bird, T. A. Lipo. "Calculating the forces created by an electrodynamic wheel using a 2-D steady-state finite-element method," IEEE Trans. Magn., Vol.44, no.3, pp. 365-372, 2008.

[6] W. Zhang, K. Zhang, et al. "Calculating Electromagnetic Force Created by Static Suspension Device Used in Permanent Magnet Electrodynamic Suspension Vehicle," Applied Computational Electromagnetics Society Journal, Vol.33, no.11, pp. 1326-1331, 2018.

[7] U. Hasirci, A. Balikci, Z. Zabar Z, et al. "3-D FEM analysis of a novel magnetic levitation system," IEEE Trans. Plasma Sci., vol45, no.5, pp. 1261-1265, 2015

[8] S. Paul, D. Bobba, et al. "Source field modeling in air using magnetic charge sheets," IEEE Trans. Magn., Vol.48, no.11, pp. 3879-3882, 2012.

[9] J. Duan, S. Xiao, et al. "A novel 3-D analytical modeling method of trapezoidal shape permanent magnet halbach array for multi-objective optimization," J Electr Eng Technol, Vol.14, no.2, pp. 635-643, 2018. 
[10] S. Paul, W. Bomela, N. Paudel, et al. "3-D Eddy Current Torque Modeling," IEEE Trans. Magn., Vol.50, no.2, pp. 905-908, 2014

[11] Y. Chen, W. Zhang, J. Z. Bird, et al. "A 3-D Analytic-Based Model of a Null-Flux Halbach Array Electrodynamic Suspension Device," IEEE Trans. Magn., Vol.51, no.11, pp. 1-5, 2015.

[12] A. Musolino, R. Rizzo, L. Sani, et al. "Null-Flux Coils in Permanent Magnets Bearings," IEEE Trans. Plasma Sci., vol45, no.7, pp. 1545-1552, 2017.
[13] U. Hasirci, A. Balikci, Z. Zabar Z, et al. "A novel magnetic-levitation system: design, implementation, and nonlinear control," IEEE Trans. Plasma Sci., vol39, no.1, pp. 492-497, 2010.

[14] J. Wright, J. Bird. "Analytic Damping and Stiffness Analysis for a 4-DOF Electrodynamic Wheel Maglev Vehicle," in Proc. ICEM. Jeju, Korea, 2018, pp. 555-561. 
